\title{
MicroRNA polymorphisms: a giant leap towards personalized medicine
}

\section{"An individual's genetic inheritance of microRNA polymorphisms associated with disease progression, prognosis and treatment holds the key to create safer and more personalized drugs and can be a giant leap towards personalized medicine."}

Voltaire, one of the greatest French authors, once said:

"Doctors are men who prescribe medicines of which they know little, to cure diseases of which they know less, in human beings of whom they know nothing" [101].

Today, 200 years after Voltaire, we have learned a great deal about many drugs, disease and the human body, but still his words seem to challenge our scientific thinking to its very core when we think of personalized medicine. Although we are able to eradicate some epidemics and claim to have cures for many of the common infectious, mental, neoplastic and cardiovascular disorders, the limitations of our current medical approaches are evident in the form of poor response rates and adverse drug reactions (ADRs).

At present, most of the pharmaceutical companies follow a 'one-size-fits-all' approach in drug development. There is a lack of reliable and costeffective ways to predict an individual's response to a drug. Worldwide use of drugs has revealed that drug response can be as diverse as the genetic background of individuals. Moreover, a poor understanding of drug-resistance in patients might result in ADRs and sometimes death. ADRs have continued to be an important and growing public health concern in the USA and worldwide $[1,2]$. In 1988, the US FDA launched the Adverse Event Reporting System (AERS) and has taken some initiatives to facilitate the integration of pharmacogenomics into drug development and clinical practice [3,102]. Because 'one drug does not fit all' we need to invest in developing a tailor-made, individualized, medicinal approach. Recently, we have learned that interindividual differences in microRNA (miRNA) regulatory pathways can influence drug response [4].

MiRNA-polymorphisms (miR-polymorphisms or miRSNPs) are a novel class of functional polymorphisms present in the human genome [4-6].
MiR-polymorphisms have been shown to influence drug response by affecting the expression of drug target genes and have been associated with many diseases such as cancer, neurological disorders and cardiovascular disorders (FIGURE 1; reviewed in [7-9]). A personalized medicinal approach using miRpolymorphisms affecting drug response among geographically and ethnically distinct individuals may hold the key to limiting ADRs in patients.

MiR-polymorphisms are a boon of the ongoing miRNA revolution. Thanks to the Human Genome Project, we now know that what was once considered as 'junk DNA', encodes evolutionary conserved miRNAs - the micromanagers of gene expression [10]. Essential for cellular and organism development, and predicted to regulate more than a third of the human genome, miRNAs are small, 21-23 nucleotide-long, independent, functional units of noncoding RNAs [11-14]. By binding to the 3'-untranslated region (UTR) of a target gene, miRNAs regulate gene expression either by inhibiting the translation of proteins and/or by destabilizing their target mRNAs $[4,15,16]$. To date, only 678 human miRNAs are known to exist, which may be only a fraction of the total in silico predictions; the total number may approach thousands or tens of thousands [17-19]. Gain or loss of miRNA function is associated with disease progression and prognosis (reviewed in [7]). Located within fragile genomic regions, miRNA genes are often deregulated in many cancers [20], such as papillary thyroid carcinoma [21], chronic lymphocytic leukemia [22,23] and breast cancer [24]. MiRNAs can potentially regulate the expression of multiple genes and pathways, are associated with disease progression and can be used in the clinic to predict drug prognosis.

Other than miRNAs, the Human Genome Project also revealed the presence of millions of polymorphisms in both coding and noncoding regions. Some of these polymorphisms are found to be present in miRNA genes and in

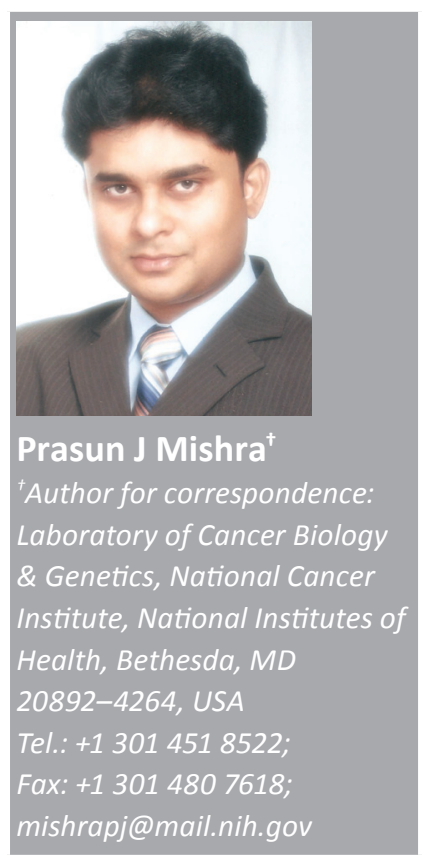

future medicine $^{\text {pits }} \mathbf{f S g}$ 


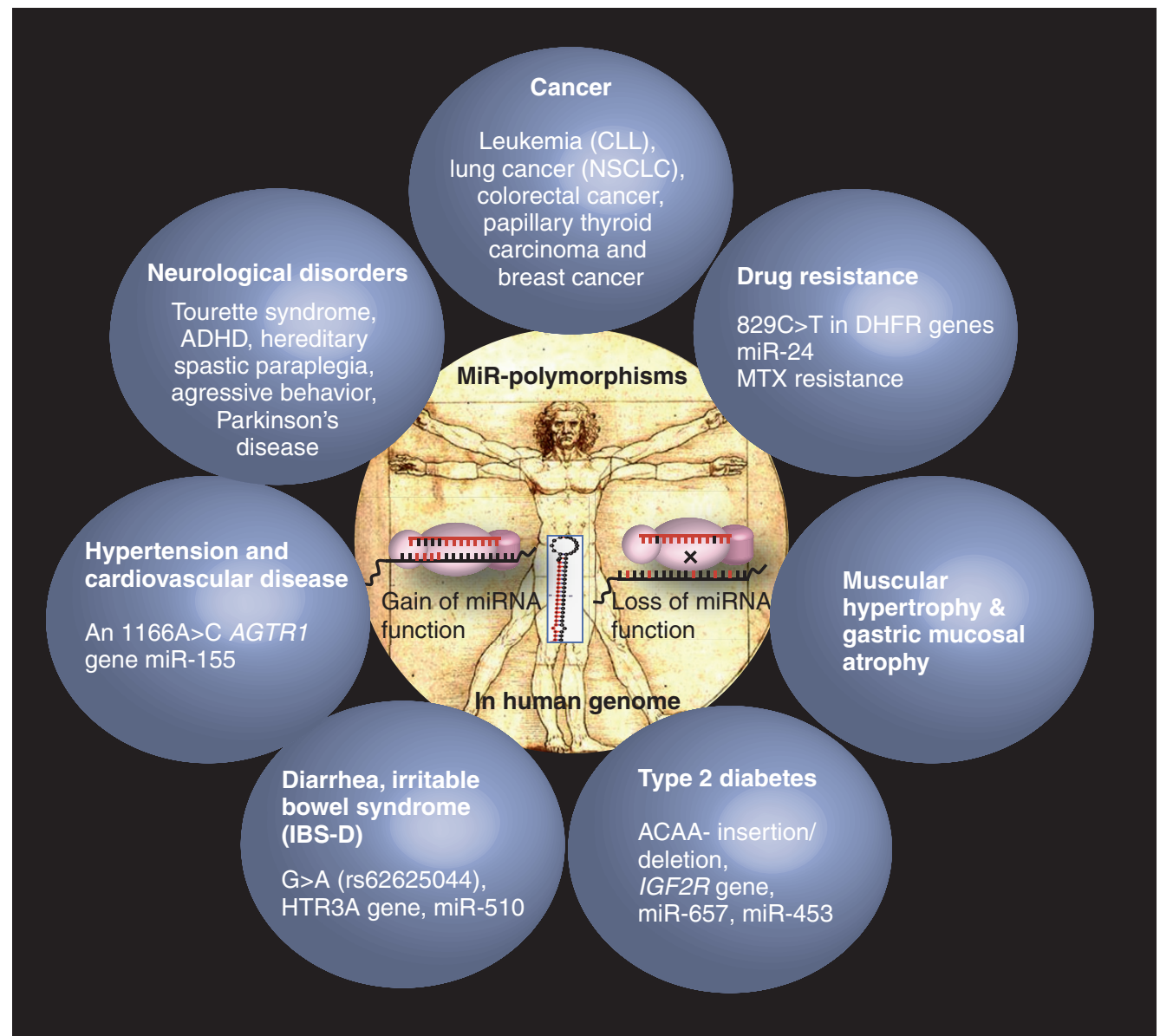

Figure 1. MiR-polymorphisms are associated with disease progression and drug response. MiR-polymorphisms are a novel class of functional polymorphisms present in the human genome. MiR-polymorphisms are found to be associated with many diseases such as cancer, neurological disorders, hypertension/cardiovascular disorders, gastric mucosal atrophy, Type II diabetes and muscular hypertrophy (reviewed in [7,8]). MiR-polymorphisms can also influence drug response $[4,6]$. MiR-polymorphisms are recently classified in three major categories: polymorphisms affecting miRNA biogenesis; at or near a miRNA target site; altering epigenetic regulation of miRNA genes (see [7]). The miRNA-target-site polymorphisms have a more gene-specific affect. MiR-polymorphisms affecting miRNA biogenesis can potentially affect expression of hundreds and thousands of genes. Associated with disease and drug response, miR-polymorphisms can be exploited for developing safer and more individualized drugs and have strong implications for personalized medicine. ADHD: Attention deficit hyperactivity disorder; AGTR1: Angiotensin receptor 1; CLL: Chronic lymphocytic leukemia; HTR3A: Serotonin receptor type 3 subunit; IBS-D: Irritable bowel syndrome; IGF2R: IGF2 receptor; miRNA: MicroRNA; MiR-polymorphisms: MicroRNA polymorphisms;

MTX: Methotrexate; NSCLC: Non-small-cell lung cancer.

The central image is adapted from The Vitruvian Man, a painting by Leonardo da Vinci [106].

the UTRs of the target genes where a miRNA binds [4]. Recent genome-wide analysis (GWA) studies of human SNPs have revealed: that several polymorphisms are present in the miRNA binding sites [25]; noncoding variations in the regulatory sites are more likely to be associated with disease than the coding region variations [26,27]; and there is a possibility that disease-associated variations may also interfere with functions of miRNAs [28]. This suggests that noncoding regions may be a hotspot for pathology, and harbor individual differences which may be exploited for tailor-made therapy.
MiR-polymorphisms have been shown to affect drug response and have the potential to confer drug resistance [7]. Recently, it was demonstrated that a $\mathrm{C}$ to $\mathrm{T}$ SNP, identified in a case-control study of childhood leukemia patients, occuring with $14.2 \%$ allelic frequency in the Japanese population, is present near the miR-24 binding site in the 3'-UTR of the dihydrofolate reductase $(D H F R)$ gene [4]. The $\mathrm{T}$ allele of the SNP results in loss of miR-24-mediated regulation of DHFR, high DHFR protein levels and confers methotrexate resistance $[4,5]$. This finding may also be useful in predicting the clinical outcome 
of methotrexate treatment [3-7]. The discovery of miR-polymorphisms has introduced 'miRNApharmacogenomics', a novel field that holds great promise for tailor-made personalized medicine. MiRNA-pharmacogenomics is the study of polymorphisms present in the miRNA regulatory pathway and its association with drug response to improve drug efficiency [3,6]. MiRNApharmacogenomics combines our knowledge in the fields of miRNA, pharmacology and genomics, to study how each individual's own genetic inheritance of miR-polymorphisms can affect the body's response to drugs, which has strong clinical implications.

Like a miRNA, a miR-polymorphism can also potentially affect expression of multiple miRNA target genes and pathways by affecting miRNA function. MiR-polymorphisms act as a double-edged sword. By creating or destroying a miRNA binding site within a target mRNA, miR-polymorphisms can result in a gain or loss of a miRNA function $[3,4,6]$. Evident from the relatively high number of miR-polymorphisms at the 3'-UTRs of target genes [25], a variant miRNA may be naturally selected [29]. Primarily, a miRNA consists of two regions, the 5'-region of a miRNA from positions $2-7$ called the 'seed' region, and the 3'-mismatch tolerant region (hereafter referred to as $3^{\prime}$-MTR) [7]. The seed region is thought to confer much of the target recognition specificity and is predicted to have less than a $1 \%$ likelihood of a polymorphism [20]. Unlike the 5'-seed region, the $3^{\prime}$-MTR is able to tolerate mismatches. Although miRNA seed region variations seem to be rare, they have the potential to affect the expression and function of the miRNA itself, and can influence expression of hundreds of genes and related pathways.

In a recent review, based on the current information within the miRNA field, we have proposed a classification of miR-polymorphisms (see [7]). There are several types of miR-polymorphisms that can exist in a cell. Polymorphisms affecting various steps of miRNA biogenesis, such as miRNA transcription, processing, export and targeting can potentially affect global biogenesis of all miRNAs with severe consequences. Polymorphisms present in pri-, pre- and mature-miRNA can potentially influence expression of hundreds of genes and pathways, broadly affecting miRNA function. Unlike polymorphisms affecting miRNA-biogenesis, miRNA target site polymorphisms have a more specific effect and are more abundant (reviewed in [7]).
MiRNA-polymorphisms were recently found to be associated with progression and prognosis of different types of cancers. Identified in some patients with familial chronic lymphocytic leukemia (CLL), a $\mathrm{C}$ to $\mathrm{T}$ polymorphism in the primary transcript of $\mathrm{miR}-15 \mathrm{a} / \mathrm{miR}-16$ was found to be associated with reduced expression of miR-15 and miR-16 [30,31]. Since 70\% of CLL cases express low levels of miR-15a/miR-16, the $\mathrm{C}$ to $\mathrm{T}$ miR-polymorphism may have implications in leukemogenesis [30-33]. In individuals with non-small-cell lung cancer (NSCLC), a significant decrease in survival was recently observed in patients that were homozygous (CC) for a pre-miR-196a2 SNP (rs11614913), suggesting that the miRSNP rs11614913 could be a prognostic marker for NSCLC [34]. More recently, a study identified rs11614913 (T to C), along with a miRSNP A to G (rs3746444) in miR-499, as being significantly associated with an increased susceptibility to breast cancer [35]. Also, a miRSNP C to T (rs93410170) in the $3^{\prime}$-UTR of estrogen receptor- $\alpha$ (ER- $\left.\alpha\right)$, was found to influence miR-206-mediated regulation of ER- $\alpha$, and was implicated in breast cancer [36]. An integrin, $\beta-4$ SNP, was suggested to influence breast tumor aggressiveness and survival [37]. A chromosomal translocation associated with human tumors, was shown to disrupt the let-7 miRNA-mediated regulation of High Mobility Group A2, and resulted in oncogenic transformation [38].

\section{"MiRNA-pharmacogenomics combines our knowledge in the fields of miRNA, pharmacology and genomics, to study how each person's own genetic inheritance of miR-polymorphisms can affect the body's response to drugs, which has strong clinical implications."}

Approximately $4.7 \%$ of papillary thyroid carcinoma (PTC) tumors have been shown to acquire a somatic mutation of rs2910164, a G/C pre-miR-146a. By affecting miR-146 expression, somatic mutation rs2910164 has been shown to be associated with the genetic predisposition to PTC [39]. Two miR-polymorphisms in the miR-221/222 and miR-146a/146b miRNA binding sites of the KIT gene were found to be associated with deregulated expression of the KIT protein, contributing to PTC [21]. Epidemiological data suggests an association of miRNA-related genetic variants to the risk of developing bladder cancer [40] and sporadic 
colorectal cancer [41]. In the 3'-UTR of the cluster of the differentiation 86 (CD86) genes, a C to $\mathrm{G}$ polymorphism (rs17281995), predicted to affect the binding of five miRNAs (miR-337, miR-582, miR-200a, miR-184 and miR-212), was significantly associated with colorectal cancer. The study also identified rs1051690 within the insulin receptor, as predicted, to affect miR-618 and miR-612 [41].

MiR-polymorphisms are associated with some neurological disorders. A var321SLITRK1 miR-polymorphism that strengthens an existing miR-189 target site in the $3^{\prime}$-UTR of the human Slit and Trk-like-1 (SLITRK1) gene was implicated in Tourette syndrome (TS) and attention deficit hyperactivity disorder. The reduced levels of SLITRK1 protein (required at high levels in the human brain for normal neurite growth) due to the miR-polymorphism might be associated with TS [42]. Implicated in hereditary spastic paraplegia patients, three different polymorphisms present in the $3^{\prime}$-UTR of receptor expression-enhancing protein 1 (REEP1) were identified in silico $[43,44]$. An A to $G$ polymorphism (rs13212041) affects miR-96-mediated regulation of the 5-hydroxytryptamine (serotonin) receptor $1 \mathrm{~B}$ (HTR1B) protein. Since deletion or down-regulation of the $H T R 1 B$ gene in mice results in aggressive behavior, individuals with ancestral A alleles exhibited more conduct-disorder behaviors than individuals with the allele $\mathrm{G}$ [45]. The polymorphism rs12720208 in the 3'-UTR of fibroblast growth factor 20 (FGF20) is identified as a risk factor for Parkinson's disease (PD). The PD-risk-allele for rs12720208 disrupts a binding site for miR-433, resulting in increased expression of FGF20, which is a risk factor for PD [46]. A 1166A to C miR-polymorphism (rs5186) results in abrogation of miR-155mediated regulation and overexpression of the angiotensin receptor 1 (AGTR1) gene. Since $A G T R 1$ overexpression results in hypertension, the miRSNP was implicated in hypertension and cardiovascular disease $[47,48]$.

MiR-polymorphisms are shown to be associated with other diseases such as diarrhea, predominant irritable bowel syndrome (IBS-D), gastric mucosal atrophy and Type 2 diabetes. A miRSNP rs62625044 G>A was shown to affect the binding of miR-510 to the serotonin receptor Type 3 subunit gene HTR3A3'-UTR, resulting in higher expression of the receptor subunit. Identified in a case-control study, rs62625044 has a strong association with female IBS-D [49]. A polymorphism of the miRNA 27 genome region was found to be associated with the development of gastric mucosal atrophy in Japanese male subjects [50]. Recently, it was demonstrated that a Type 2 diabetes-associated ACAA-insertion/deletion polymorphism is a miR-polymorphism that results in the loss of miR-657-mediated regulation of human insulin-like growth factor 2 receptor (IGF2R) [51]. Hence, a polymorphic difference in the inherited DNA sequence of individuals that affects function of a miRNA can have a profound effect and is responsible for disease progression, prognosis and can affect drug response.

\section{"MiR-polymorphisms are emerging as a powerful tool for studying the biology of disease and will aid in its diagnosis and prognosis."}

Although we have identified and validated some key miR-polymorphisms in the past 2 years, many more still need to be identified and characterized. As great acts are often made up of small deeds [103], identification and validation of miR-polymorphisms associated with human disease will revolutionalize the field of personalized medicine. With recent advances in human genome research, this might not be a difficult task. Due to its affordability, more families are now interested in the genome sequencing of a child either at its birth or later in life. In May 2008, the US Congress passed the US Genetic Information and Nondiscrimination Act to legally protect the misuse of an individual's genetic information [104,105]. This is one more positive step towards personalized medicine whose need is absolute and urgent to prevent ADRs. Approximately 2.2 million hospitalized patients suffered from ADRs and 106,000 deaths were reported yearly [1]. Identifying and characterizing disease-specific miR-polymorphisms may be one more step towards avoiding ADRs.

In conclusion, by affecting miRNA function, miR-polymorphisms can affect the expression of hundreds of genes and related pathways in a cell. MiR-polymorphisms are emerging as a powerful tool for studying the biology of disease and will aid in its diagnosis and prognosis. MiRNA-pharmacogenomics, with the knowledge of functional miR-polymorphisms, may provide a better understanding of pharmacological responses to a drug and will revolutionize drug discovery and the developmental process. A better understanding of the role of 
miR-polymorphism in drug response will allow the development of a more personalized and/or a population-specific treatment, providing greater degrees of success. Hence, an individual's genetic inheritance of miR-polymorphisms associated with disease progression, prognosis and treatment holds the key to create safer and more personalized drugs, and can be a giant leap towards personalized medicine.

\section{Acknowledgements}

The author thanks Dr Glenn Merlino, Laboratory of Cancer Biology and Genetics, and Dr Rita Humeniuk,
Laboratory of Cellular Oncology, at the National Cancer

Institute, NIH for the critical review of the manuscript.

\section{Financial \& competing interests disclosure}

The author has no relevant affliations or financial involvement with any organization or entity with a financial interest in or financial conflict with the subject matter or materials discussed in the manuscript. This includes employment, consultancies, honoraria, stock ownership or options, expert testimony, grants or patents received or pending, or royalties.

No writing assistance was utilized in the production of this manuscript.

\section{Bibliography}

Papers of special note have been highlighted as:

" of interest

"EI of considerable interest

1 Lazarou J, Pomeranz BH, Corey PN: Incidence of adverse drug reactions in hospitalized patients: a meta-analysis of prospective studies. JAMA 279, 1200-1205 (1998).

2 Moore TJ, Cohen MR, Furberg CD: Serious adverse drug events reported to the Food and Drug Administration, 19982005. Arch. Intern. Med. 167, 1752-1759 (2007).

3 Bertino JR, Banerjee D, Mishra PJ: Pharmacogenomics of microRNA: a miRSNP towards individualized therapy. Pharmacogenomics 8, 1625 (2007).

-1* This editorial first introduced the microRNA-pharmacogenomics field.

4 Mishra PJ, Humeniuk R, Longo-Sorbello GS, Banerjee D, Bertino JR: A miR-24 microRNA binding-site polymorphism in dihydrofolate reductase gene leads to methotrexate resistance. Proc. Natl Acad. Sci. USA 104, 13513-13518 (2007).

"* Role of a microRNA-polymorphism (miR-polymorphism) in drug resistance; the term miRSNP was coined and defined.

5 Mishra PJ, Banerjee D, Bertino JR: A microRNA binding site polymorphism in dihydrofolate reductase gene leads to methotrexate resistance. AACR Meeting April 2007. Proc. Am. Assoc. Cancer Res. 48, 4516, 1070 (2007).

6 Mishra PJ, Mishra PJ, Banerjee D, Bertino JR: MiRSNPs or miR-polymorphisms, new players in microRNA mediated regulation of the cell: introducing microRNA pharmacogenomics. Cell Cycle 7, 853-858 (2008).

- ${ }^{-}$Coined and defined the term miR-polymorphism.
7 Mishra PJ, Bertino J: MicroRNA polymorphisms: the future of pharmacogenomics, molecular epidemiology and individualized medicine. Pharmacogenomics 10(3), 399-416 (2009).

=- Recent review on miR-polymorphisms, classified miR-polymorphisms.

8 Sethupathy P, Collins FS: MicroRNA target site polymorphisms and human disease. Trends Genet. 24(10), 489-497 (2008).

= Opinion on the role of miRNA target site poymorphisms in human disease.

9 Chen K, Song F, Calin G, Wei Q, Hao X, Zhang W: Polymorphisms in microRNA targets: a gold mine for molecular epidemiology. Carcinogenesis 29(7), 1306-1311 (2008).

- Review about miRNA target site polymorphisms.

10 Bartel DP, Chen CZ: Micromanagers of gene expression: the potentially widespread influence of metazoan microRNAs. Nat. Rev. Genet. 5, 396-400 (2004).

11 Lagos-Quintana M, Rauhut R, Lendeckel W, Tuschl T: Identification of novel genes coding for small expressed RNAs. Science 294, 853-858 (2001).

- Landmark paper that identified miRNA genes.

12 Lau NC, Lim LP, Weinstein EG, Bartel DP: An abundant class of tiny RNAs with probable regulatory roles in Caenorhabditis elegans. Science 294, 858-862 (2001).

- Landmark paper that identified miRNA genes.

13 Lee RC, Ambros V: An extensive class of small RNAs in Caenorhabditis elegans. Science 294, 862-864 (2001)

- $\quad$ Landmark paper that identified miRNA genes.
14 Yanaihara N, Caplen N, Bowman E et al: Unique microRNA molecular profiles in lung cancer diagnosis and prognosis. Cancer Cell 9 , 189-198 (2006).

15 Selbach M, Schwanhausser B, Thierfelder N, Fang Z, Khanin R, Rajewsky N: Widespread changes in protein synthesis induced by microRNAs. Nature 455, 58-63 (2008).

- Established that miRNA destabilization is one of the principle mechanisms of action of an miRNA.

16 Baek D, Villen J, Shin C, Camargo FD, Gygi SP, Bartel DP: The impact of microRNAs on protein output. Nature 455 , 64-71 (2008).

- Established that miRNA not only fine-tunes protein output at the translational level but also degrades mRNAs.

17 Berezikov E, Guryev V, van de Belt J, Wienholds E, Plasterk RH, Cuppen E: Phylogenetic shadowing and computational identification of human microRNA genes. Cell 120, 21-24 (2005)

18 Griffiths-Jones S, Saini HK, van Dongen S, Enright AJ: MiRBase: tools for microRNA genomics. Nucleic Acids Res. 36, D154-D158 (2008).

19 Rigoutsos I, Huynh T, Miranda K, Tsirigos A, McHardy A, Platt D: Short blocks from the noncoding parts of the human genome have instances within nearly all known genes and relate to biological processes. Proc. Natl Acad. Sci. USA 103, 6605-6610 (2006).

20 Didiano D, Hobert O: Perfect seed pairing is not a generally reliable predictor for miRNA-target interactions. Nat. Struct. Mol. Biol. 13, 849-851 (2006).

- MiRNA seed region cannot reliably predict miRNA-RNA target interactions. 
$21 \mathrm{He} \mathrm{H}$, Jazdzewski K, Li W et al:: The role of microRNA genes in papillary thyroid carcinoma. Proc. Natl Acad. Sci. USA 102, 19075-19080 (2005).

22 He L, He X, Lowe SW, Hannon GJ: MicroRNAs join the p53 network - another piece in the tumour-suppression puzzle. Nat. Rev. Cancer 7, 819-822 (2007).

23 Calin GA, Garzon R, Cimmino A, Fabbri M, Croce CM: MicroRNAs and leukemias: how strong is the connection? Leuk. Res. 30, 653-655 (2006).

24 Iorio MV, Ferracin M, Liu CG et al.: MicroRNA gene expression deregulation in human breast cancer. Cancer Res. 65, 7065-7070 (2005)

25 Saunders MA, Liang H, Li WH: Human polymorphism at microRNAs and microRNA target sites. Proc. Natl Acad. Sci. USA 104, 3300-3305 (2007)

26 Manolio TA, Brooks LD, Collins FS: A HapMap harvest of insights into the genetics of common disease. J. Clin. Invest. 118, 1590-1605 (2008).

27 Wellcome Trust Case Control Consortium: Genome-wide association study of 14,000 cases of seven common diseases and 3000 shared controls. Nature 447, 661-678 (2007).

28 Glinsky GV: An SNP-guided microRNA map of fifteen common human disorders identifies a consensus disease phenocode aiming at principal components of the nuclear import pathway. Cell Cycle 7, 2570-2583 (2008).

29 Chen K, Rajewsky N: Natural selection on human microRNA binding sites inferred from SNP data. Nat. Genet. 38, 1452-1456 (2006).

30 Calin GA, Ferracin M, Cimmino A et al.: A microRNA signature associated with prognosis and progression in chronic lymphocytic leukemia. N. Engl. J. Med. 353, 1793-1801 (2005).

"- MiR-15a/miR-16 pre-miRNA polymorphism associated with chronic lymphocytic leukemia (CLL).

31 Raveche ES, Salerno E, Scaglione BJ et al.: Abnormal microRNA-16 locus with synteny to human 13q14 linked to CLL in NZB mice. Blood 109, 5079-5086 (2007).

"- MiR-15a/miR-16 pre-miRNA polymorphism associated with CLL.

32 Calin GA, Dumitru CD, Shimizu M et al.: Frequent deletions and down-regulation of microRNA genes miR15 and miR16 at 13q14 in chronic lymphocytic leukemia. Proc. Natl Acad. Sci. USA 99, 15524-15529 (2002).

" MiR-15a/miR-16 pre-miRNA polymorphism associated with CLL.
33 Calin GA, Liu CG, Sevignani C et al: MicroRNA profiling reveals distinct signatures in B cell chronic lymphocytic leukemias. Proc. Natl Acad. Sci. USA 101, 11755-11760 (2004).

- MiR-15a/miR-16 pre-miRNA polymorphism associated with CLL.

$34 \mathrm{Hu} \mathrm{Z}$, Chen J, Tian T et al.: Genetic variants of miRNA sequences and non-small cell lung cancer survival. J. Clin. Invest. 118, 2600-2608 (2008).

"- rs11614913 is associated with non-small-cell lung cancer.

35 Hu Z, Liang J, Wang Z et al.: Common genetic variants in pre-microRNAs were associated with increased risk of breast cancer in Chinese women. Hum. Mutat. 30(1), 79-84 (2008).

36 Adams BD, Furneaux H, White BA: The micro-ribonucleic acid (miRNA) miR-206 targets the human estrogen receptor- $\alpha(E R \alpha)$ and represses $E R \alpha$ messenger RNA and protein expression in breast cancer cell lines. Mol. Endocrinol. 21, 1132-1147 (2007).

"* A miRSNP $(\mathrm{C}>\mathrm{T}$, rs93410170) is associated with breast cancer.

37 Brendle A, Lei H, A Brandt et al.: Polymorphisms in predicted microRNA binding sites in integrin genes and breast cancer: ITGB 4 as prognostic marker. Carcinogenesis 29(7), 1394-1399 (2008).

38 Mayr C, Hemann MT, Bartel DP: Disrupting the pairing between let-7 and Hmga2 enhances oncogenic transformation. Science 315, 1576-1579 (2007).

"* Characterized a translocation that affects let7-mediated regulation of oncogene $H M G a 2$.

39 Jazdzewski K, Murray EL, Franssila K, Jarzab B, Schoenberg DR, de la Chapelle A: Common SNP in pre-miR-146a decreases mature miR expression and predisposes to papillary thyroid carcinoma. Proc. Natl Acad. Sci. USA 105, 7269-7274 (2008).

40 Yang H, Dinney CP, Ye Y, Zhu Y, Grossman HB, Wu X: Evaluation of genetic variants in microRNA-related genes and risk of bladder cancer. Cancer Res. 68, 2530-2537 (2008).

41 Landi D, Gemignani F, Naccarati A et al.: Polymorphisms within micro-RNA-binding sites and risk of sporadic colorectal cancer. Carcinogenesis 29, 579-584 (2008).

42 Abelson JF, Kwan KY, O'Roak BJ et al: Sequence variants in SLITRK1 are associated with Tourette's syndrome. Science 310, 317-320 (2005).

"- A miR-polymorphism (var321-SLITRK1) is associated with Tourette's syndrome.
43 Zuchner S, Wang G, Tran-Viet KN et al: : Mutations in the novel mitochondrial protein REEP1 cause hereditary spastic paraplegia Type 31. Am. J. Hum. Genet. 79, 365-369 (2006).

- Identified miR-polymorphisms in REEP1 3'-UTR in silico.

44 Beetz C, Schule R, Deconinck T et al.: REEP1 mutation spectrum and genotype/ phenotype correlation in hereditary spastic paraplegia Type 31. Brain 131, 1078-1086 (2008).

- Identified miR-polymorphisms in REEPI 3'-UTR in silico.

45 Jensen KP, Covault J, Conner TS, Tennen H, Kranzler HR, Furneaux HM: A common polymorphism in serotonin receptor $1 \mathrm{~B}$ mRNA moderates regulation by miR-96 and associates with aggressive human behaviors. Mol. Psychiatry (2008) (Epub ahead of print).

= $\mathrm{A}>\mathrm{G}(\mathrm{rs} 13212041)$ has a role in aggressive behavior.

46 Wang G, van der Walt JM, Mayhew G et al.: Variation in the miRNA-433 binding site of FGF20 confers risk for Parkinson disease by overexpression of $\alpha$-synuclein. Am. J. Hum. Genet. 82, 283-289 (2008).

47 Martin MM, Buckenberger JA, Jiang J et al.: The human angiotensin II type 1 receptor +1166 A/C polymorphism attenuates microrna-155 binding. J. Biol. Chem. 282, 24262-24269 (2007).

=1166A $>\mathrm{C}(\mathrm{rs} 5186)$ is associated with hypertension.

48 Sethupathy P, Borel C, Gagnebin M et al.: Human microRNA-155 on chromosome 21 differentially interacts with its polymorphic target in the AGTR1 3' untranslated region: a mechanism for functional singlenucleotide polymorphisms related to phenotypes. Am. J. Hum. Genet. 81, 405-413 (2007)

-1166A $>\mathrm{C}(\mathrm{rs} 5186)$ is associated with hypertension.

49 Kapeller J, Houghton LA, Monnikes $\mathrm{H}$ et al.: First evidence for an association of a functional variant in the microRNA-510 target site of the serotonin receptor-type $3 \mathrm{E}$ gene with diarrhea predominant irritable bowel syndrome. Hum. Mol. Genet. 17, 2967-2977 (2008).

"\# A miRSNP $(G>A$, rs62625044) is asssociated with irritable bowel syndrome.

50 Arisawa T, Tahara T, Shibata T et al.: A polymorphism of microRNA 27a genome region is associated with the development of gastric mucosal atrophy in Japanese male subjects. Dig. Dis. Sci. 52, 1691-1697 (2007). 
51 Lv K, Guo Y, Zhang Y, Wang K, Jia Y, Sun S: Allele-specific targeting of hsa-miR-657 to human IGF2R creates a potential mechanism underlying the association of ACAAinsertion/deletion polymorphism with Type 2 diabetes. Biochem. Biophys. Res. Commun. 374, 101-105 (2008).

- A miR-polymorphism ACAA insertion/deletion is associated with Type II diabetes.

\section{Websites}

101 Medicine quotes http://thinkexist.com/quotations/medicine/

102 US Food and Drug Administration, Adverse events reporting system description http://www.fda.gov/cder/aers/default.htm

103 Lao Tzu Quotes http://en.thinkexist.com/quotation/great_ acts_are_made_up_of_small_deeds/214524. html
104 Genetic Information Nondiscrimination Act of 2008 http://www.govtrack.us/congress/billtext. xpd?bill $=$ h110 $-493 \&$ show - changes $=0$ \&pagecommand $=$ print

105 National Human Genome Research Institute http://www.genome.gov/10002077

106 A Vitruvian Man by Leonardo da Vinci http://upload.wikimedia.org/wikipedia/ commons/2/22/Da_Vinci_Vitruve_Luc_ Viatour.jpg 